\title{
Delirium awareness - Improving recognition and management through education and use of a care pathway
}

\author{
RUBEN TAURO
}

Ulster Hospital, South Eastern Health and Social Care Trust, Northern Ireland

\begin{abstract}
Delirium occurs in $10-20 \%$ of medical patients on admission and a further $10-30 \%$ develop delirium as an inpatient. Delirium is associated with increased length of stay, morbidity, mortality, and risk of institutional placement. There is poor knowledge of delirium recognition and management, and a need to raise awareness and training of all staff. NICE have produced guidelines for diagnosis, prevention and management of delirium.

A retrospective departmental audit demonstrated that delirium was under-recognised i.e. only $5.7 \%$ of discharges in one year. A staff questionnaire revealed poor knowledge of types of delirium and a significant underestimation of prevalence, with poor identification of risk factors. A multi-professional group was formed to raise staff awareness and develop a care pathway for delirium. A 19 bed acute elderly care ward was identified for the project.
\end{abstract}

Ward based and departmental educational meetings were held. A Trust based awareness programme was also provided. Information leaflets on delirium were produced for patients, carers and families and posters at ward level. Environmental changes (signage) at ward level were introduced to improve the environment for patients. A delirium care pathway was created to encourage documentation of mental score, assessment of delirium, review of reversible medical causes and a nursing care plan. This pathway was reviewed regularly on ward rounds and feedback given to staff present.

Following these interventions the notes of 106 consecutive discharges were reviewed over an 11 -week period. $99 \%$ of at risk patients were screened for delirium. $35 \%$ of patients were diagnosed with delirium increasing the recognition rate from $5.7 \%$. There was significant improvement among the staff in recognizing and managing patients with delirium through the use of a delirium care pathway. Education improves understanding and awareness of delirium and a care pathway focuses attention on this area, improving patient safety and quality of care.

\section{Problem}

Delirium (sometimes called 'acute confusional state') is a common clinical syndrome characterised by disturbed consciousness, cognitive function or perception, which has an acute onset and fluctuating course. It usually develops over $1-2$ days (1). It is a serious condition that is associated with poor outcomes. However, it can be prevented and treated if dealt with urgently (1). The Ulster Hospital, South Eastern Health and Social Care Trust (SEHSCT), Northern Ireland, is a district general teaching hospital with approximately 440 beds providing care in a range of medical and surgical specialities. There are four wards dedicated to Care of the Elderly. Older people and people with dementia, severe illness or a hip fracture are more at risk of delirium (1).

\section{Background}

Delirium occurs in $10-20 \%$ of medical patients on admission and a further $10-30 \%$ develop delirium as an inpatient (2). Delirium is associated with increased length of stay, morbidity, mortality and risk of institutional placement $(3,4,5)$. There is poor knowledge of delirium recognition and management and a need to raise awareness among staff. NICE have produced guidelines for diagnosis, prevention and management of delirium and a delirium awareness workshop session guide designed to raise staff awareness (1)

Major difficulties arise when introducing evidence and clinical guidelines into routine daily practice (6). Substantial evidence suggests that to change behaviour is possible, but this change generally requires comprehensive approaches at different levels (doctor, team practice, hospital, wider environment), tailored to specific settings and target groups (6).

\section{Baseline Measurement}

The Ulster Hospital, South Eastern Health and Social Care Trust (SEHSCT), Northern Ireland, is a district general teaching hospital with approximately 440 beds providing care in a range of medical and surgical specialties. There are four wards ( 78 beds) dedicated to acute Elderly Care which includes a 20 bedded all age stroke unit.

A previous retrospective audit of discharges from the Dept. of Elderly Care demonstrated that delirium was under-recognised i.e. 
only 5.7\% of discharges April 2011 - March 2012 recorded a diagnosis of delirium. A staff questionnaire was subsequently performed to assess the understanding and educational needs of staff about delirium. This revealed poor knowledge of types of delirium and a significant underestimation of prevalence with poor identification of risk factors. It was noted that the majority of staff, particularly allied health professionals and nursing colleagues, had received no previous teaching on delirium.

\section{Design}

We aimed to prevent delirium in patients identified at risk and when delirium develops improve our recognition and management through use of a delirium care pathway. A delirium project group (medical, nursing and pharmacy) was formed. A 19 bedded acute elderly care ward identified for the project. The results and proposed plans for change were presented at the safety, quality and experience (SQE) programme within the Trust and disseminated to different staff groups through ward and departmental based educational meetings. Ward managers were engaged in providing information leaflets to the patients, carers and families. The medical team were involved in monitoring compliance with risk assessment and delirium care pathways.

\section{Strategy}

Improvement (PDSA) cycle 1

Assessment of the problem through a staff questionnaire revealed a gap in the knowledge of staff in recognising and managing patients with delirium. It was noted that the majority of staff (particularly non medical staff) had received no previous teaching on delirium. As a result, ward based thirty minute educational sessions for nursing staff were held twice monthly. Delirium was included as a topic on four sessions and topics extended to other areas relevant to Elderly Care. Departmental educational sessions (one hour for junior medical staff) and one hour for interdisciplinary education (i.e. physiotherapy, occupational therapy, social worker, nursing, medical) identified. A trust based awareness programme was provided through the old age psychiatry team (two, two hour sessions per 6 months). These educational sessions used information from the NICE delirium awareness workshop session guide (1). Information leaflets on delirium were produced for patients, carers and families and posters at ward level. The information leaflets briefly explained what delirium is, how people with delirium behave, who are at risk, what makes it worse, and what makes it better. Also the leaflets advised the relatives to notify a member of the staff if there was a change in behaviour. If relatives wanted further information, contact details of liaison mental health services for older people was printed on the leaflets. Signage at ward level was introduced to improve the environment for patients.

Improvement (PDSA) cycle 2

A delirium care pathway was created to encourage documentation of mental score, assessment of delirium through use of the confusion assessment method (CAM) (7). Patients with delirium had a review of reversible medical causes and a nursing care plan documented as part of the pathway. This pathway was reviewed regularly on Consultant ward rounds and feedback given to nursing and medical staff present on the round. This educational programme and care pathway was introduced over a 6 month period. Subsequently a retrospective audit to look at recognition and management of delirium using the care pathway was undertaken.

\section{Results}

The notes of 106 consecutive discharges were reviewed over an 11 -week period at the end of the study period. $99 \%$ of at risk patients were screened for delirium. There was limitation in using mental score for patients who were agitated or had language and hearing difficulties. Confusion assessment method (CAM) was used in $82 \%$ of patients. Medical and nursing management checklist was completed in $94 \%$ of CAM positive patients. $35 \%$ of patients were diagnosed with delirium increasing the recognition rate from the previous audit of $5.7 \%$.

\section{Lessons and Limitations}

A process of staff education and introduction of a delirium care pathway has increased delirium awareness within the elderly care staff. Our view is that both components of the interventionprogramme were required to improve delirium awareness. A limitation of this data is that we cannot determine if one strategy alone was responsible for the improvement. Education of staff was undertaken at multiple levels within the Trust and we believe this is important as delirium is relevant to most acute medical and surgical specialties. A limitation of the ward based sessions is that only small numbers of staff were able to attend any one session making them labour intensive. The care pathway demonstrated clear evidence of improved assessment and management of risk factors for delirium. The pathway was regularly reviewed on ward rounds and we believe this improved compliance. Our improvements have been demonstrated in one ward area and we aim to role this out across the unit. We anticipate that a 'champion' will be required in each ward area to continue to promote education at ward level and challenge compliance with the care pathway. We would suggest that this should be either a member of the permanent medical or nursing staff complement of the ward to provide continuity of education and learning.

\section{Conclusion}

Education improves understanding and awareness of delirium and a care pathway focuses attention on this area, improving patient safety and quality of care.

\section{References}

1. NICE guideline 103. Delirium: diagnosis, prevention and management 2010

2. Nayeem K, O’Keefe S. Delirium. Clinical Medicine 2003; 3: 


\section{BMJ Quality Improvement Reports}

412-415.

3. Van ZyI LT and Seitz DP. Delirium: Concisely: Condition is associated with increased morbidity, mortality and length of hospitalisation.Geriatrics 2006; 61:18-22.

4. O'Keefe, S \& Lavan, J. Prognostic significance of delirium in older patients. Journal of the American Geriatrics Society, 45, 174-178.

5. Potter J, George J. The prevention, diagnosis and management of delirium in older people: concise guidelines. Clinical Medicine 2006; 6 303-308.

6. Grol R, Grimshaw J. From best evidence to best practice: effective implementation of change in patients' care. Lancet. 2003;362(9391):1225-30

7. Laurila JV, Pitkala KH, Strandberg TE, Tilvis RS. Confusion assessment method in the diagnostics of delirium among aged hospital patients: would it serve better in screening than a diagnostic instrument? Int J Geriatr Psychiatry. 2002 Dec;17(12):1112-9

\section{Declaration of interests}

None

\section{Acknowledgements}

Dr April Heaney, Mentor and Care of Elderly Consultant

Dr Donna Lennon, Specialty Doctor in Elderly Care

Karen Hull, Ward sister

Nursing and Allied health Staff of Ward 22 